\title{
An overview on the antioxidant, anti-inflammatory, antimicrobial and anti-cancer activity of grape extract
}

\author{
Sara Karami, Maryam Rahimi* and Arash Babaei \\ Department of Biology, Faculty of Sciences, Malayer University, Malayer, Iran
}

\begin{abstract}
The dietary consumption of grape and its products is associated with a lower incidence of degenerative diseases such as cardiovascular disease and certain types of cancers. Most recent interest has focused on the bioactive phenolic compounds in grape. Anthocyanins, flavanols, flavanols and resveratrol are the most important grape polyphenols because they possess many biological activities such as antioxidant, cardioprotective, anticancer, anti-inflammation, antiaging and antimicrobial properties. Grape-related anti-tumoral activity encompasses a wide array of biological mechanisms and cellular targets, eventually leading to inhibition of cell growth and to enhanced apoptosis in several cancer cell lines, including lung, colon, breast, bladder, leukemia and prostate tumors. Those effects are likely modulated at the molecular level through selectively modulating the redox balance and displaying anti-oxidant as well as pro-oxidant actions. GSE-related anti-cancer activity mostly relies on the induced increase in reactive oxygen species, followed by the orchestrated down- and up-regulation of several key-molecular pathways, including MAPK kinases, PI3K/Akt, NF-kB, cytoskeleton proteins and metalloproteinases. Promising results obtained in vitro as well as on animal studies suggest that GSE may have a great relevance as source of potential new pharmacological molecules, and could represent an important opportunity for clinical research.
\end{abstract}

\section{Introduction}

Grape is one of the most economically important plant species due to its diverse uses in production of wine, grape juice and other food products [1-3]. It is cultivated in all continents in the temperate regions where sufficient rain, warm and dry summers as well as relatively mild winters are normal climatic patterns [2-4]. The qualities of grape products are characterized by their metabolic compositions [5,6]. Grape is a phenol-rich plant, and these phenolics are mainly distributed in the skin, stem, leaf and seed of grape, rather than their juicy middle sections. Total concentration of phenolic compounds was about $2178.8,374.6$, 23.8 , and $351.6 \mathrm{mg} / \mathrm{g}$ GAE (gallic acid equivalent) in seed, skin, flesh, and leaf, respectively [6-8]. The total phenolic content of grape skins varied with cultivar, soil composition, climate, geographic origin, and cultivation practices or exposure to diseases, such as fungal infections [9]. Flavonoids represent a widespread and common group of natural polyphenols produced by the phenylpropanoid pathway. They confer UV-protection, determine flower coloration, attract pollinators, and act as tissue protectors in case of pathogen attack or oxidative damage. In grapes, flavonoids are primarily located in the epidermal layer of berry skin and the seeds. Flavonoids are the main groups of soluble phenolics in grapes as well as major contributors of the biological activities in products derived from grapes [10,11]. Flavonoids in red wine are the most feasible phytochemicals, responsible for this phenomenon. Flavonoids have cardioprotective, antioxidant, anti-inflammatory, anticancer and antimicrobial properties, and are one of the most potent nutraceuticals in food and phytopharmaceutical products $[11,12]$. Therefore, it is paramount to understand the principles of biological activity, bioavailability and metabolism of grape flavonoids in relation to human health. ${ }^{4}$ Grape seeds represent a high percentage (20\% to $26 \%$ ) of the grape marc obtained as a byproduct from white winemaking and keep a vast proportion of grape polyphenols [4,9]. Grape seeds represent a low percentage of the fruit weight (about $5 \%$ on average), although the antioxidant phenolic compounds present in the seeds account for
$60 \%-70 \%$ of the total polyphenols in the grape [10]. Polyphenols are the most important phytochemicals in grape because they possess many biological activities and health-promoting benefits. The phenolic compounds mainly include anthocyanins, flavanols, flavonols, stilbenes (resveratrol) and phenolic acids. Anthocyanins are pigments, and mainly exist in grape skins. Flavonoids are widely distributed in grapes, especially in seeds and stems, and principally contain (+)-catechins, $(-)$-epicatechin and procyanidin polymers. Anthocyanins are the main polyphenolics in red grapes, while flavan-3-ols are more abundant in white varieties. The reported evidences of beneficial health effects of phenolic compounds include inhibiting some degenerative diseases, such as cardiovascular diseases, and certain types of cancers, reducing plasma oxidation stress and slowing aging [11]. Phenolic compounds are also regarded as preservatives against microbes and oxidation for food. What's more, in vivo assays showed that phenolic compounds are bioavailable [13].

\section{Flavonoids in grapes}

Flavonoids represent a large family of secondary metabolites and nearly 6000 structures have been identified in plants. The diversity in their chemical structures contributes to their broad range of physiological and biological activities. The most common flavonoids found in grapes are anthocyanins(3-O-monoglucosides

${ }^{*}$ Correspondence to: Maryam Rahimi, Department of Biology, Faculty of Sciences, Malayer University, Malayer, Iran, Tel: +98 813333 9841; E-mail: m.rahimi@malayeru.ac.ir

Key words: grape extract, polyphenols, anti-oxidant, anti-inflammatory, anticancer

Received: March 02, 2018; Accepted: March 21, 2018; Published: March 26, 2018 
or 3,5-O-diglucosides of malvidin, cyanidin, peonidin, delphinidin, pelargonidin and petunidin, as well as their acetyl-, p-coumaroyland/or caffeoyl-esters), flavonols (3-O-glycosides of quercetin , kaempferol , myricetin, laricitrin, isorhamnetinandsyringetin), flavanols $[(+)$-catechin, $(-)$-epicatechin , (-)-epicatechin-3-O-gallate], dihydroflavonols (astilbin and engeletin) and proanthocyanidins .Anthocyanins are found only in red grape varieties [14,15]. They accumulate mainly in the berry skin, but in some varieties known as "teinturier" (or dyed), anthocyanin pigments are found to accumulate in the flesh of the berry. It is important to note that there is a close correlation between anthocyanin biosynthesis and berry development: it starts at "veraison" when proanthocyanidin biosynthesis is concluded and reaches maximum level at berry "maturity". Each grape species and variety respectively has a unique set of anthocyanins. European grapes produced only anthocyanidins3-O-monoglucosides, whereas muscadine grapes produced only anthocyanidins 3,5-O-diglucosides [15].

\section{Antioxidant action}

As a result of their aerobic metabolism, active cells produce toxic byproducts known as reactive oxygen species (ROS). ROS account for a wide range of aggressive free radicals produced by various metabolite pathways in living cells $[2,16]$. Under normal physiological conditions, ROS are important regulatory agents in the complex signaling network of cells. They play a major role in promoting cell growth and differentiation, adaptation to metabolic and physiological stresses, immune response as well as protection from pathogen invasion. However, several factors may cause an over-accumulation of ROS by interrupting regular cellular processes and thus exposing tissues to conditions of oxidative stress $[13,17]$. When cells are exposed to oxidative stress they easily undergo oxidative damage that leads to a cascade of degenerative processes. Development of severe pathologies including neurodegenerative diseases, diabetes, cancer, liver diseases, cardiovascular diseases, and rapid aging are among the possible outcomes. It is generally assumed that therapeutic treatment with antioxidants is the most effective way to control oxidative stress and to avoid occurrence of oxidative damage. Various molecules by weight and structure including enzymes and hormones may have antioxidant activities in biological systems. Antioxidants may neutralize oxidative stress in various ways including inhibition of free radical formation (preventive antioxidants), interrupting autoxidation chain reactions (chain breaking antioxidants), up-regulating and protecting cellular antioxidant defenses mechanisms (indirect antioxidants), neutralizing the action of metal pro-oxidant ions (metal chelators),inhibiting the action of pro-oxidative enzymes (enzyme inhibitors) and increasing the activities of other antioxidants (synergistic compounds) [13]. Flavonoids represent a large group of low molecular weight compounds with high antioxidant properties. Their specific chemical structure allows them to reduce oxidative stress through numerous mechanisms. For example, it was reported that, in vitro, flavonoids could act both as preventive antioxidants and chain breaking antioxidants [scavenging superoxide, peroxyl, alkoxyl and hydroxyl radicals as well as preventing low-density lipoprotein (LDL) oxidation. On the other hand, flavonoids can also act as metal chelators (reducing ferric and cupric ions), and enzyme inhibitors (inhibiting the enzymes involved in ROS generation: xanthine oxidase; protein kinase C; cyclooxygenase; lipoxygenase; glutathione S-transferase; microsomal monooxygenase; mitochondrial succinoxidase and NADH oxidase) [13,17]. However, in vivo research has also demonstrated that flavonoids also may act as indirect antioxidants by up-regulating antioxidant defense system and increasing uric acid concentration in the plasma. Therefore, because of their metabolic conversion in the human body, flavonoids generate large amounts of simple phenolic acids, which have significant effects in scavenging free radicals and improving the action of other antioxidants $[18,19]$.

\section{Anti-inflammatory action}

Inflammation is a protective response of tissues against cell injury, irritation, pathogen invasions, as well as mechanism for eliminating damaged and necrotic cells. Several environmental stress factors may cause inflammation. Under normal physiological conditions, a short period of acute inflammation can overcome negative effects on injured tissue. However, if inflammation is prolonged, chronic inflammation can have developed. Chronic inflammation is the main mediator in the development of chronic diseases such as cancer, Alzheimer's, neurodegenerative diseases, cardiovascular diseases, diabetes, arthritis, autoimmune and pulmonary diseases [15]. When signal-dependent transcription factors [nuclear factor $\kappa \mathrm{B}(\mathrm{NF}-\kappa \mathrm{B})$ and activating protein 1 (AP-1)] are activated, they induce the expression of genes involved in inflammatory response. Intensive production and secretion of pro-inflammatory cytokines and chemokines, once started, can form concentration gradients in affected tissues, which may lead to the amplification of the initial inflammatory response. As a result, additional immune cells are recruited, and increased levels of ROS are produced. Under normal physiological conditions, anti-inflammatory cytokines act as immuno regulators to control the inflammatory reactions. Deregulation of precise control mechanism of inflammation leads to chronic inflammation and promotion of chronic disease $[20,21]$. Grape polyphenols have been shown to decrease chronic inflammation either by modulation of inflammatory pathways or by reducing ROS levels. As natural compounds, grape flavonoids and proanthocyanidins can target multiple pathways to overcome chronic inflammation, and thus are more effective compared to synthetic monotargeted anti-inflammatory drugs. Freeze-dried extract of wine from "Jacquez" grapes, which contains mainly flavonoids, anthocyanins, proanthocyanidins and hydroxycinnamic acid derivatives, showed higher anti-inflammatory activity when compared to the commercial non-steroidal anti-inflammatory drug(NSAID) indomethacin. It has also been demonstrated that proanthocyanidins in grape seeds have high anti-inflammatory action, because they scavenge free radicals, prevent lipid peroxidation and inhibit formation of pro-inflammatory [21]. Proanthocyanidins extracted from the grape seeds have also been found to have an immune-modulatory role in inflammatory conditions that exert an overproduction of nitric oxide and prostaglandin E2 $[22,23]$. The suppression effect of extracts obtained by red and white grape pomaces on chronic inflammation induced by lipopolysaccharide and galactosamine, has been investigated in vivo. The authors found that the extract of red grape pomace suppresses the activation of inflammatory transcription factor NF- $\kappa B$ and thus could be used as raw material for both extraction of new anti-inflammatory candidates or as an additive in processing functional foods [15].

\section{Antimicrobial and antiviral activities}

Antimicrobial activities of grape, wine and grape-derived byproducts have been widely discussed. It has been demonstrated that grape seed extract of V. vinifera "Bangalore Blue Grapes" exhibits stronger antibacterial activity against Gram-positive bacteria compared to the response from Gram-negative bacteria. Grape juice, skin and seed extracts from black table grapes "Ribier" var. have also been found to have a strong inhibitory effect against the growth of Listeria 
monocytogenes. In addition, research has also shown that red colored (anthocyanin pigments) grape juice and skin extract have $\mathrm{pH}$-dependent anti-listerial activity, while the seed extract showed $\mathrm{pH}$-independent anti-listerial activity $[11,13]$. Strong anti-listerial activities have also been demonstrated for extracts of grape berries, seeds, pomace, and stems from red and white grape varieties. The main active compounds were catechine, epicatechine and epicatechine gallate [22,23].

\section{Anticancer properties of grape}

Grape antioxidants have drawn an increased attention for their potential anticancer effects [24]. Several studies suggest that the high consumption of grape components could be associated with the reduced risk of certain cancers such as breast cancer and colon cancer [13,25]. The anticancer effects of grape antioxidants have been demonstrated in in vitro and in vivo models. Grape antioxidants have been shown to induce cell cycle arrest and apoptosis in cancer cells as well as prevent carcinogenesis and cancer progression in rodent models $[25,26]$. Considering the diversity of grape antioxidants, it is very likely that these compounds are to exert potential anticancer activity by acting on multiple cellular events associated with tumor initiation, promotion, and progression. Proposed mechanisms of potential anticancer effects of grape antioxidants include antioxidant, anti-inflammatory, and antiproliferative activities [27]. Grape antioxidants could act as free radical's scavengers, and chelating agents help to reduce physiological reactive oxygen species (ROS). ROS is known as an important mediator of apoptosis since initiation and regulation of apoptosis is associated with modifications in the oxidative environment $[13,28]$.

\section{Conclusion}

Grape antioxidants especially grape seed proanthocyanidins (GSPs) show very promising inhibitory effects on a variety of cancer cells. In vitro treatment of NSCLC cells (A549, H1299, H460, H226, and H157) with GSPs resulted in significant growth inhibition and induction of apoptosis, which were associated with the inhibitory effects of GSPs. Grape seed extract (GSE) was also found to selectively inhibit the growth and cause cell cycle arrest and apoptotic death in both Detroit 562 and FaDu HNSCC cells by activating DNA damage checkpoint cascade, including ataxia telangiectasia mutated/ataxia telangiectasia-Rad3- related-checkpoint kinase 1/2cell division cycle $25 \mathrm{C}$ as well as caspases 8,9 , and 3 . In addition, GSE caused accumulation of intracellular ROS was identified as a major mechanism of its effect for growth inhibition, DNA damage and apoptosis, which was remarkably reversed by antioxidant $\mathrm{N}$-acetylcysteine. ${ }^{22}$ In summary, with respect to cancer prevention and treatment, grape seed extracts or its proanthocyanidins have received most investigation for their potential anticancer activities. Some studies show very promising potential of GSE as an anticancer agent. However, most available reports are focusing on their in vitro activities and mechanisms. Since the absorption of these phenolic compounds is limited, the dose responses of GSE and its components need to be determined in animals and humans as well as its in vivo mechanisms. The potential use of grape skin and seed extracts in cancer prevention has great potential, and further investigation into this exciting field is warranted.

\section{References}

1. Pezzuto JM (2008) Grapes and human health: a perspective. J Agric Food Chem 56: 6777-6784. [Crossref]
2. Hertog MG, Kromhout D, Aravanis C, Blackburn H, Buzina R, et al. (1995) Flavonoid intake and long-term risk of coronary heart disease and cancer in the seven countries study. Arch Intern Med 155: 381-386. [Crossref]

3. Vidavalur R, Otani H, Singal PK, Maulik N (2006) Significance of wine and resveratrol in cardiovascular disease: French paradox revisited. Exp Clin Cardiol 11: 217-225. [Crossref]

4. Shrikhande AJ (2000) Wine by-products with health benefits. Food Res Internat 33: 469-474

5. Silva RC, Rigaud J, Cheynier V, Chemina A (1991) Procyanidin dimers and trimers from grape seeds. Phytochemistry 30: 1259-1264.

6. Wada M, Kido H, Ohyama K, Ichibangas T, Kishikaw N, et al. (2007) Chemiluminescen screening of quenching effects of natural colorants against reactive oxygen species: evaluation of grape seed, monascus, gardenia and red radish extracts as multi-functional food additives. Food Chem 101: 980-986.

7. Dopico-Garcia MS, Fique A, Guerra L, Afonso JM, Pereira O, et al. (2008) Principal components of phenolics to characterize red Vinho Verde grapes: anthocyanins or noncoloured compounds? Talanta 75: 1190-1202.

8. Spacil Z, Novakova L, Solich P (2008) Analysis of phenolic compounds by high performance liquidchromatography and ultra-performance liquid chromatography. Talanta 76: 189-199. [Crossref]

9. Bagchi D, Bagchi M, Stohs SJ, Das DK, Ray CA, et al. (2000) Free radicals and grape seed proanthocyanidin extract: importance in human health and disease prevention. Toxicology 148: 187-197. [Crossref]

10. Cantos E, Espin JC, Tomas-Barberan FA (2002) Varietal differences among the polyphenol profiles of seven table grape cultivars studied by LC-DAD-MS-MS. J Agric Food Chem 50: 5691-5696. [Crossref]

11. Xia EQ, Deng GF, Guo YJ, Li HB (2010) Biological activities of polyphenols from grapes. Int J Mol Sci 11: 622-646. [Crossref]

12. Maier T, Schieber A, Kammerer DR, Carle R (2009) Residues of grape (Vitis vinifera L.) seed oil production as a valuable source of phenolic antioxidants. Food Chem 112: 551-559.

13. Georgiev V, Ananga A, Tsolova V (2014) Recent Advances and Uses of Grape Flavonoids as Nutraceuticals. Nutrients 6: 391-415. [Crossref]

14. Chacona MR, Ceperuelo-Mallafrea V, Maymo-Masipa E, Mateo-Sanzb JM, Arolac L, et al. (2009) Grape-seed procyanidins modulate inflammation on human differentiated adipocytes in vitro. Cytokine 47: 137-142. [Crossref]

15. Zhou K, Raffoul JJ (2012) Potential anticancer properties of grape antioxidants. $J$ Oncol 2012: 803294. [Crossref]

16. Novaka I, Janeiroa P, Serugab M, Oliveira-Brett AM (2008) Ultrasound extracted flavonoids from four varieties of Portuguese red grape skins determined by reversephase high-performance liquid chromatography with electrochemical detection. Anal Chim Acta 630: 107-115. [Crossref]

17. Ali K1, Maltese F, Choi YH, Verpoorte R (2010) Metabolic constituents of grapevine and grape-derived products. Phytochem Rev 9: 357-378. [Crossref]

18. Laurent C, Besancon P, Caporiccio B (2007) Flavonoids from a grape seed extract interact with digestive secretions and intestinal cells as assessed in an in vitro digestion/ Caco-2 cell culture model. Food Chem 100: 1704-1712.

19. Wenzel U, Kuntz S, Brendel MD, Daniel H (2000) Dietary flavone is a potent apoptosis inducer in human colon carcinoma cells. Cancer Res 60: 3823-3831. [Crossref]

20. Garcia-Jares C, Vazquez A, Lamas JP, Pajaro M, Alvarez-Casas M (2015) Antioxidan White Grape Seed Phenolics: Pressurized Liquid Extracts from Different Varieties. Antioxidants (Basel) 4: 737-749. [Crossref]

21. Ghafoor K, Choi YH, Jeon JY, Jo IH (2009) Optimization of ultrasound-Assisted extraction of phenolic compounds, antioxidants, and anthocyanins from grape (Vitis vinifera) seeds. J Agric Food Chem 57: 4988-4994. [Crossref]

22. De la Cerda-Carrasco A, López-Solís R, Núñez-Kalasic H, Peña-Neira A, ObrequeSlier E (2015) Phenolic composition and antioxidant capacity of pomaces from four grape varieties (Vitis vinífera L.) J Sci Food Agric 95: 1521-15275.

23. Ribéreau-Gayon P, Glories Y, Maujean A, Dubordieu D (2006) Handbook of Enology John Wiley \& Sons Ltd.; Chichester, UK.

24. Briviba K, Pan L, Rechkemmer G (2002) Red wine polyphenols inhibit the growth of colon carcinoma cells and modulate the activation pattern of mitogen-activated protein kinases. J Nutr 132: 2814-2818. [Crossref] 
25. Tyagi A, Agarwal R, Agarwal C (2003) Grape seed extract inhibits EGF induced and constitutively active mitogenic signaling but activates JNK in human prostate carcinoma DU145 cells: possible role in antiproliferation and apoptosis. Oncogene 22: 1302-1316. [Crossref]

26. Glade MJ (1999) Food, nutrition, and the prevention of cancer: a globa perspective. American Institute for Cancer Research/World Cancer Research Fund, American Institute for Cancer Research. Nutrition 15: 523-526. [Crossref]
27. Yang CS, Landau JM, Huang MT, Newmark HL (2001) Inhibition of carcinogenesis by dietary polyphenolic compounds. Annu Rev Nutr 21: 381-406. [Crossref]

28. Gao N, Budhraja A, Cheng S, Yao H, Zhang Z, et al. (2009) Induction of Apoptosis in Human Leukemia Cells by Grape Seed Extract Occurs via Activation of c-Jun NH2Terminal Kinase. Clin Cancer Res 15: 140-149.

Copyright: ( 2018 Karami S. This is an open-access article distributed under the terms of the Creative Commons Attribution License, which permits unrestricted use, distribution, and reproduction in any medium, provided the original author and source are credited. 